\title{
"One-sample concept" micro-combinatory for high throughput TEM of binary films
}

\author{
György Sáfrán \\ Institute for Technical Physics and Materials Science, Centre for Energy Research, Hungarian Academy of Sciences, H.A.S. Konkoly-Thege Miklós út 29-33., H-1121 Budapest, Hungary
}

\section{ARTICLE INFO}

Keywords:

Single specimen combinatory

Binary system

High throughput TEM

Gradient film

\begin{abstract}
A B S T R A C T
Phases of thin films may remarkably differ from that of bulk. Unlike to the comprehensive data files of Binary Phase Diagrams [1] available for bulk, complete phase maps for thin binary layers do not exist. This is due to both the diverse metastable, non-equilibrium or instable phases feasible in thin films and the required volume of characterization work with analytical techniques like TEM, SAED and EDS. The aim of the present work was to develop a method that remarkably facilitates the TEM study of the diverse binary phases of thin films, or the creation of phase maps. A micro-combinatorial method was worked out that enables both preparation and study of a gradient two-component film within a single TEM specimen. For a demonstration of the technique thin $\mathrm{Mn}_{x} \mathrm{Al}_{1-x}$ binary samples with evolving concentration from $x=0$ to $x=1$ have been prepared so that the transition from pure $\mathrm{Mn}$ to pure $\mathrm{Al}$ covers a $1.5 \mathrm{~mm}$ long track within the $3 \mathrm{~mm}$ diameter TEM grid.

The proposed method enables the preparation and study of thin combinatorial samples including all feasible phases as a function of composition or other deposition parameters. Contrary to known "combinatorial chemistry", in which a series of different samples are deposited in one run, and investigated, one at a time, the present micro-combinatorial method produces a single specimen condensing a complete library of a binary system that can be studied, efficiently, within a single TEM session. That provides extremely high throughput for TEM characterization of composition-dependent phases, exploration of new materials, or the construction of phase diagrams of binary films.
\end{abstract}

\section{Introduction}

Combinatorial techniques are very effective in the study of the correlations of preparation parameters, e.g. composition and physical properties of diverse materials. Combinatory is widely used in chemistry, biology and pharmaceutical innovation. In materials science, "multiple-sample concept" was suggested by Hanak in 1970 [2] who, instead of searching for new materials by studying only one composition at a time, proposed combinatorial solutions. The first examples for combinatorial preparation of samples for microscopy investigations were published in the 1990s and thence, the development of the field has a continuous interest. Schultz and co-workers [3] applied computer aided mask movement to prepare samples of various compositions at a density of 10,000 sample/in ${ }^{2}$. Roskov et al. [4] used combinatorial technique to deposit polymer samples onto individual TEM grids, so that each sample exhibited different composition. Rack et al. [5] used DC magnetron sputtering from up to 4 targets to deposit combinatorial layers spread over a surface of several $\mathrm{cm}$-size and applied a computer simulation to predict the film composition. Julthongpiput et al. [6] prepared a series of organosilane specimens with a gradual change from hydrophobic to hydrophilic and the samples were investigated by contact angle measurements, atomic force microscopy, and automated optical microscopy. Olk and Haddad [7] applied combinatorial deposition of 64 discrete samples from up to four sputtering targets to systematically control the microstructure of $\mathrm{Al}_{x} \mathrm{Si}_{(1-x)}$ alloys through variations in composition and growth temperature. The microstructure, surface morphology and phase distribution were investigated using X-ray diffraction and atomic force microscopy techniques.

Regarding efficiency, the above techniques represent partial solutions: the way of preparation is combinatorial, the investigation, however, has to be carried out on a number of individual samples, one at a time. Barna and co-workers [8, 9] applied an experimental arrangement in which a sample with variable composition was both deposited and investigated within a single TEM grid: A shield with a $1 \mathrm{~mm}$ diameter aperture was fixed above the grid so that a pair of evaporation sources was facing the substrate at different angles, through the aper-

Email address: safran@mfa.kfki.hu (G. Sáfrán) 
ture. As an alternative solution to aperture a small platelet was fixed edge-on at the center of the TEM grid. As a result the grown films showed spatially varying composition at the area of half-shadow of the aperture, or of the platelet.

The method $[8,9]$ represents a "one-sample concept", however, suffers from fundamental flaws: (i) the concentration profile is unintentional (ii) the area of variable composition is very short, (iii) it does not cover the entire (0-100\%) range and (iiii) poor reproducibility. These failures cause positioning of a wished composition go beyond control, inhibit both a clear separation of the various phases and a revelation of the whole set of data, therefore, impedes the method to spread.

The aim of the present work was to develop a one-sample technique that corrects the incompleteness of the above solutions. The so-called "micro-combinatory" ( $\mu$-combinatory) introduced in this paper is known to be the first that enables both preparation and investigation of a combinatorial binary specimen within a single TEM grid, that covers the entire $(0-100 \%)$ composition range and exhibits a programmed concentration profile providing clear separation and easy positioning of the composition-dependent phases. Thanks to its all-in-one feature, such a $\mu$-combinatorial sample enables a high throughput TEM study of a complete library of phases of a binary system. For a demonstration of the method thin $\mu$-combinatorial MnAl samples will be discussed here. MnAl is of recent technological interest [10] due to its tetragonal phase with hard magnetic properties.

\section{Experimental}

The principles of the preparation of a $\mu$-combinatorial sample are as follows:

$\mathrm{A}_{x} \mathrm{~B}_{1-x}$ sample with gradient composition, in the range of $0 \leq x \leq 1$, is deposited along a track that fits a $3 \mathrm{~mm}$ diameter TEM grid. This is implemented by means of a device [11] that actuates a shutter with a $0.2 \times 1 \mathrm{~mm}^{2}$ slot in fine steps above the TEM grid meanwhile the powers of the two DC magnetron sources, "A" and "B", are regulated in sync with the propagation of the slot: the fluence of component A gradually decreases from its $100 \%$ to $0 \%$, and that of B changes inversely, from $0 \%$ to $100 \%$ (a dependence of the fluence on the output power was calibrated, previously, for both sources).

A typical sample appears as a $1 \mathrm{~mm}$ wide and $2.5 \mathrm{~mm}$ long layer track including a gradient section of $1.5 \mathrm{~mm}$ in length that is enclosed in between $0.5 \mathrm{~mm}$ long sections of exclusively A and B materials, respectively.

The arrangement for deposition and the build-up of the $\mu$-combinatorial TEM specimen are illustrated in Fig. 1. The three sections of the layer track deposited on the grid are indicated in the scheme: A on the left and B on the right represent sections of $100 \%$ component $\mathrm{A}$ and $100 \%$ component B component, respectively and in between them, A ? B represents the gradient section. This built up of the sample makes possible the TEM characterization of both the binary phases of variable compositions and the phases of the constituting (A and $\mathrm{B}$ ) materials.

The deposition of $\mathrm{Mn}-\mathrm{Al} \mu$-combinatorial samples was carried out as follows:

The sampleswere deposited on TEM grids covered with either carbon, or $\mathrm{SiO}_{\mathrm{x}}$ foil: The vacuum system was evacuated with a turbo molecular pump to a base pressure of $5 \times 10^{-6} \mathrm{~Pa}$. The substrates were mounted in the $\mu$-combinatorial device and load-locked into the chamber. Ar sputtering gas was introduced to the system at a partial pressure of $2.5 \times 10^{-1} \mathrm{~Pa}$. Both targets were cleaned behind closed shutters by pre-sputtering with their $100 \%$ power for 5 min. Based on previous calibrations the maximum (100\%) power values for the $\mathrm{Mn}$ and $\mathrm{Al}$ sources were selected to be $225 \mathrm{~W}$ and $150 \mathrm{~W}$, respectively that pro-
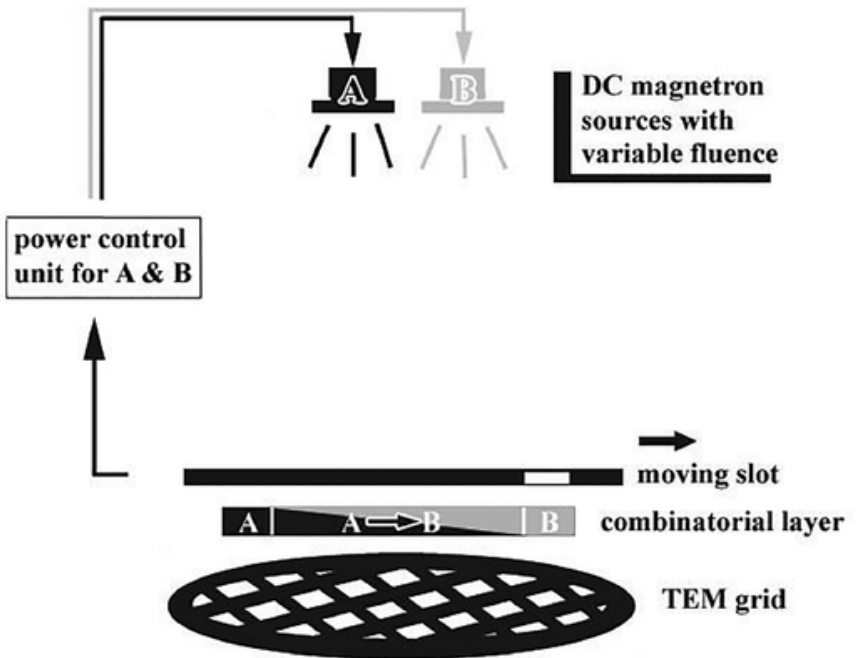

Fig. 1. Scheme of the principle of micro-combinatorial deposition: components A and B are deposited by DC magnetron sputtering onto a TEM grid through a moving slot. The output powers of the sources "A" and "B" are adjusted in accordance with the position of the slot along the grid. The track of a thin micro-combinatorial layer is deposited in $3 \mathrm{sec}-$ tions: pure A, gradient section of A ? B and pure B that are 0.5, 1.5 and $0.5 \mathrm{~mm}$ long, respectively.

vided equal fluencies. Subsequently, the motion of the slot of the $\mu$-combinatorial device was started (and maintained during the experiment), the shutter of the Mn source was opened and Mn was deposited through the moving slot onto the TEM grid. This provided the pure Mn section of the combinatorial layer track. In due time the power of the $\mathrm{Al}$ source was reduced to zero and its shutter was opened. It was followed by the gradual increase of the power of the Al source from 0 to $100 \%$, simultaneously, with the decrease of the power of the Mn source from 100 to $0 \%$ and as a result the bimetallic, gradient section of the track was deposited. As the power of the Mn source arrived to zero and, simultaneously, that of the $\mathrm{Al}$ source arrived to its maximum, the Mn source was switched off and the pure Al section of the track was deposited. Finally, the shutter of $\mathrm{Al}$ source was closed, the source was switched off and the actuation of the slot was halted.

In order to prevent Mn-containing samples for oxidation a topping layer of amorphous Si-oxide was used that protected the sample for a remarkably long period, as well, during annealing [12].

The $\mu$-combinatorial samples, prepared as above, were characterized by a $200 \mathrm{kV}$ Philips CM 20 TEM and a $300 \mathrm{kV}$ JEOL 3010 HREM. For a clear positioning within the sample the combinatorial layer track was aligned with the x-axis of the stage of the TEM. The local composition along the track was measured by a Bruker QX 200 Energy Dispersive X-ray Spectrometer (EDS) attached to the TEM, under conditions of elevation angle: $13^{\circ}$, sample tilting angle: $20^{\circ}$ and measured area: $\sim 5 \mu \mathrm{m}$ diameter. For quantification standardless analysis by the Cliff-Lorimer calculation was applied using the $\mathrm{Al} \mathrm{K} \alpha$ and $\mathrm{Mn} \mathrm{K} \alpha$ peaks with the sensitivity factors $k_{\mathrm{Al}}=0.985$ and $k_{\mathrm{Mn}}=1.350$. The structure and morphology was investigated by bright and dark field imaging and selected area electron diffraction (SAED). The evaluation of the SAED patterns was carried out with the help of the software "Process Diffraction" developed by Lábár [13]. It is to mention that only the peak positions of the Pdf database were used for identification of phases, the aspects of peak intensities were not considered.

\section{Results and discussion}

The photo of a TEM grid with a $\mathrm{Mn}-\mathrm{Al} \mu$-combinatorial sample DC sputter-deposited onto carbon foil by the method described above is shown in Fig. 2. The dimensions $\left(2.5 \times 1 \mathrm{~mm}^{2}\right)$ of the combinatorial 


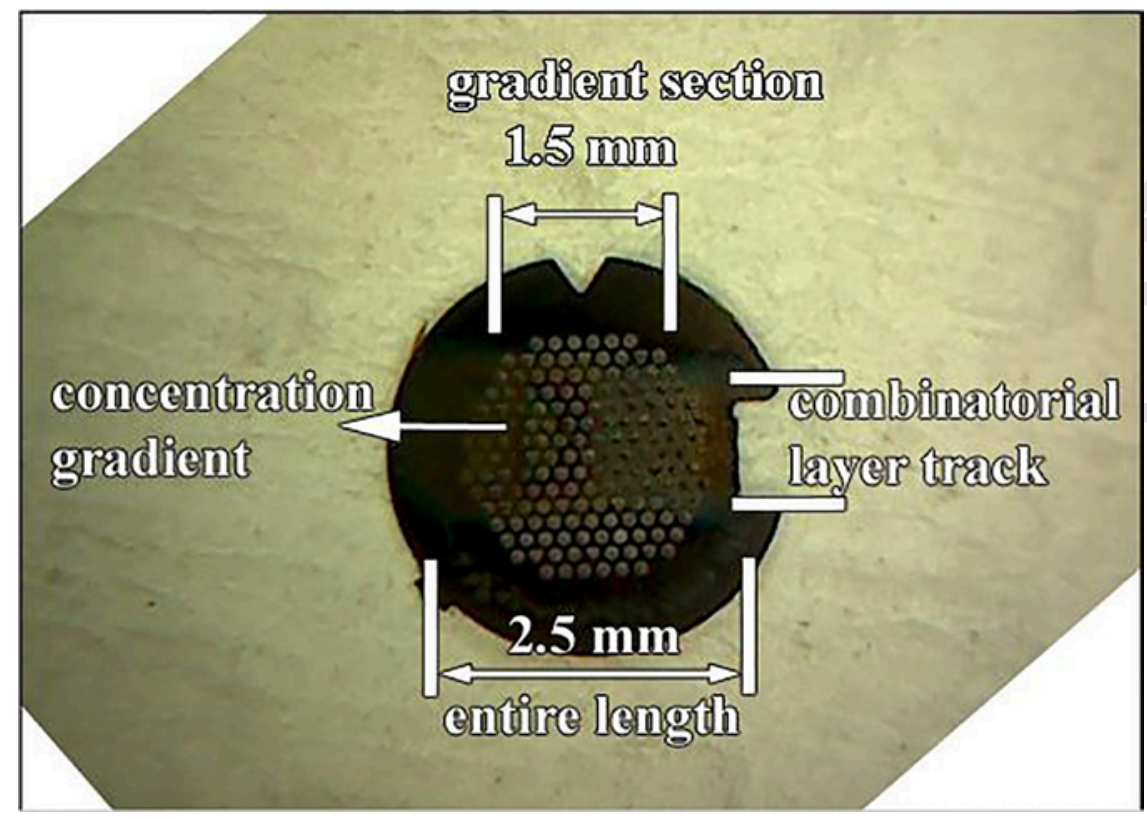

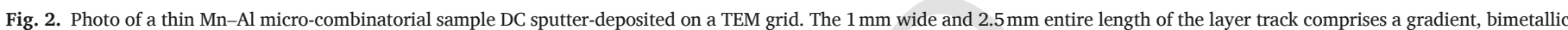
section of $1.5 \mathrm{~mm}$ length enclosed by sections of $0.5 \mathrm{~mm}$ long pure $\mathrm{Mn}$ and pure $\mathrm{Al}$, respectively.

track, including the $1.5 \times 1 \mathrm{~mm}$ size gradient section, fit well to the view-field of the microscope and enables detailed structural and morphological characterization of all possible compositions.

It is to note that Mo grid was used in this experiment suitable for post deposition annealing. Its hexagonal mesh was set for deposition so that the grid bars were aligned at $60^{\circ}$ with respect to the combinatorial track line; in case of rectangular mesh e.g. standard $\mathrm{Cu}$ grid, however, $45^{\circ}$ azimuth is advised. The diagonal alignment ensures that if, at a certain place, a given composition is masked out by a grid bar, it will, definitely, be available in a couple of sideward meshes within the $1 \mathrm{~mm}$ wide track. At the present sample the concentration gradient is $0.067 \%$ per $\mu \mathrm{m}$ i.e. $1 \%$ per $15 \mu \mathrm{m}$. Therefore, it is straightforward to locate a certain composition within the sample by counting the bars of the TEM grid at low magnification. The refining of the selection is carried out at higher magnifications, by checking the local composition by EDS (or EELS) microanalysis. The morphology and structure of the layer, at the selected composition, can be characterized by TEM imaging and electron diffraction methods.

The elemental concentration profile of the Mn-Al sample shown in Fig. 2 was measured by EDS as a function of the position along the combinatorial track. The EDS profiles shown in Fig. 3 represent linear

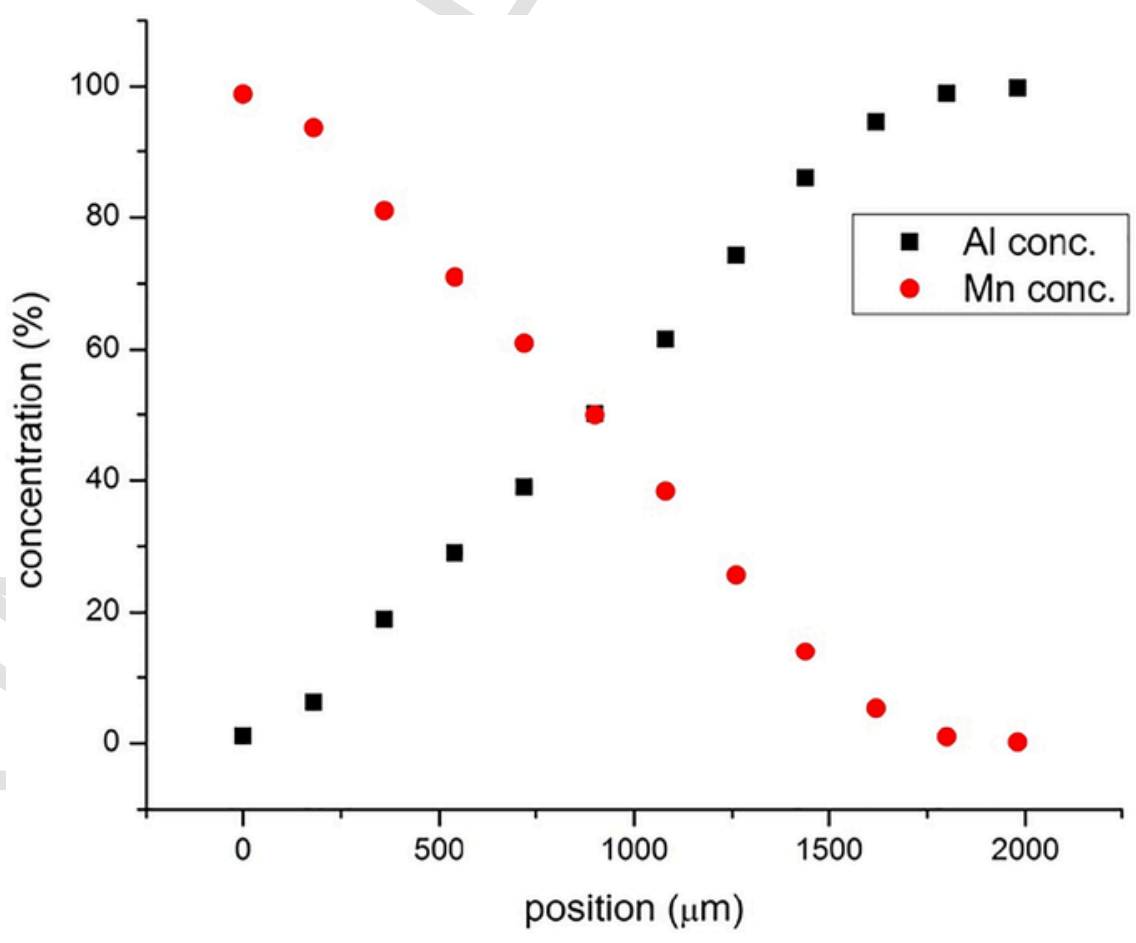

Fig. 3. Mn and $\mathrm{Al}$ concentration profiles of the micro-combinatorial sample shown in Fig 2 measured by EDS as a function of the position along the TEM grid. 
concentration variation of the components along the gradient section, except a rounding at the ends, i.e. at the transitions between bimetallic and mono-metallic. The two short, non-linear parts are attributed to a number of factors: (i) the width of the slot is finite; (ii) the magnetron sources face the slot at an inclined angle and (iii) there is a $0.5 \mathrm{~mm}$ gap between substrate and slot. A detailed characterization of the mechanisms causing the non-linear sections is out of focus of the present paper. However, it is straightforward to determine the local composition within the non-linear sections, since the concentration can be checked by EDS at any place of interest.

Microstructures revealed at selected compositions of a Mn-Al $\mu$-combinatorial sample are shown in Fig. 4. The bright field TEM micrographs in Fig. 4(a), (b), (c) represent areas of the as deposited sample at $90 \%, 40 \%$ and $20 \% \mathrm{Mn}$ contents, respectively, while that in (d) an area of $40 \% \mathrm{Mn}$ is shown after heat treatment at $500 \mathrm{C} / 0.5 \mathrm{~h}$ in $\mathrm{Ar}-\mathrm{H}_{2}$ mixture. The insets are the corresponding SAED patterns and dark field (DF) images. The DF images were taken with a $20 \mu \mathrm{m}$ size objective aperture positioned over the first intense ring or group of rings of the SAEDs (Mn $330+\mathrm{MnO} 111$ in (a), Mn 330 in (b), Mn $330+\mathrm{Al}$ 111 in (c) and $\mathrm{Mn} 330+\mathrm{Mn} 332+200 \mathrm{MnAl}$ in (d). It was revealed that the as-deposited binary layer, regardless of the composition, exhibits fine-grained $\alpha$-Mn phase, (Pdf 32-0637) depicted by the 330 ring marked in all the SAEDs of Fig. 4. In addition to $\alpha$-Mn cubic MnO (Pdf
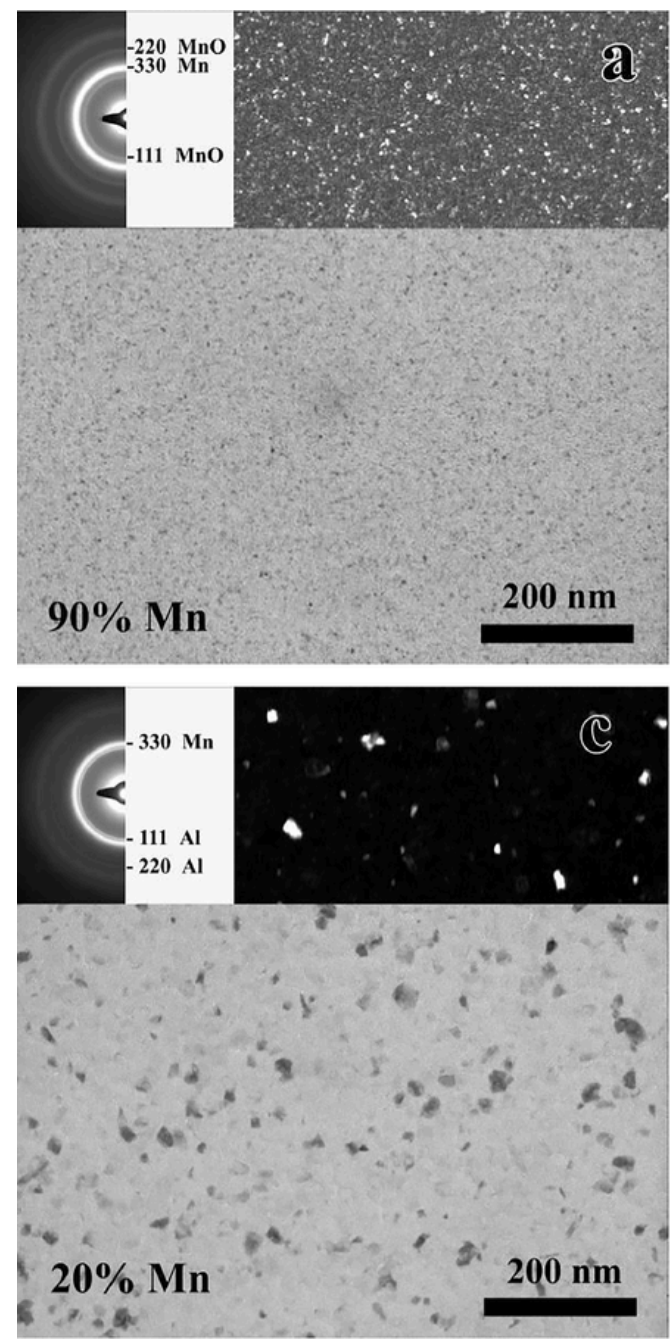

75-0621) is present, at high Mn concentrations, in the form of nm-size particles, as shown by the BF, DF images and SAED of (a). Oxide formation occurred, in spite of the applied $\mathrm{SiO}_{\mathrm{x}}$ coating, because the sample was brought to air for a short time prior to $\mathrm{SiO}_{\mathrm{x}}$ deposition. As soon as the Mn content in the gradient sample drops below 40\% the Mn-oxide diminishes as demonstrated in (b). It was found that aluminum is completely dissolved in $\alpha$-Mn up to high concentrations and forms distinct fcc $\mathrm{Al}$ phase only above $80 \%$ content as denoted by both $\mathrm{Al}$ crystallites in the BF and DF images and 111 and 200 lines of $\mathrm{Al}$ (Pdf 04-0787) in the SAED of (c). Beside the issue of oxidation the layer with $40 \%$ $\mathrm{Mn}$ content is interesting also for another reason: a tetragonal $\mathrm{Mn}_{14} \mathrm{Al}_{11}$ phase (Pdf 11-0520) appears in the film upon annealing at 500C/0.5 h in $\mathrm{Ar}-\mathrm{H}_{2}$ mixture that is in (d) represented by the BF and DF images and the appearance of 001, 110 and 200 rings of the phase in the SAED inset.

Detailed TEM analysis is out of the focus of the present paper, the examples above, however, may illustrate that $\mu$-combinatory is fully adapted for a comprehensive study of a binary layer system.

\subsection{Features and field of application}

Micro-combinatory, beside TEM, may facilitate high throughput characterization of binary systems by further analytical techniques e.g. nano-indentation, Kelvin probe, scanning electron microscopy, and
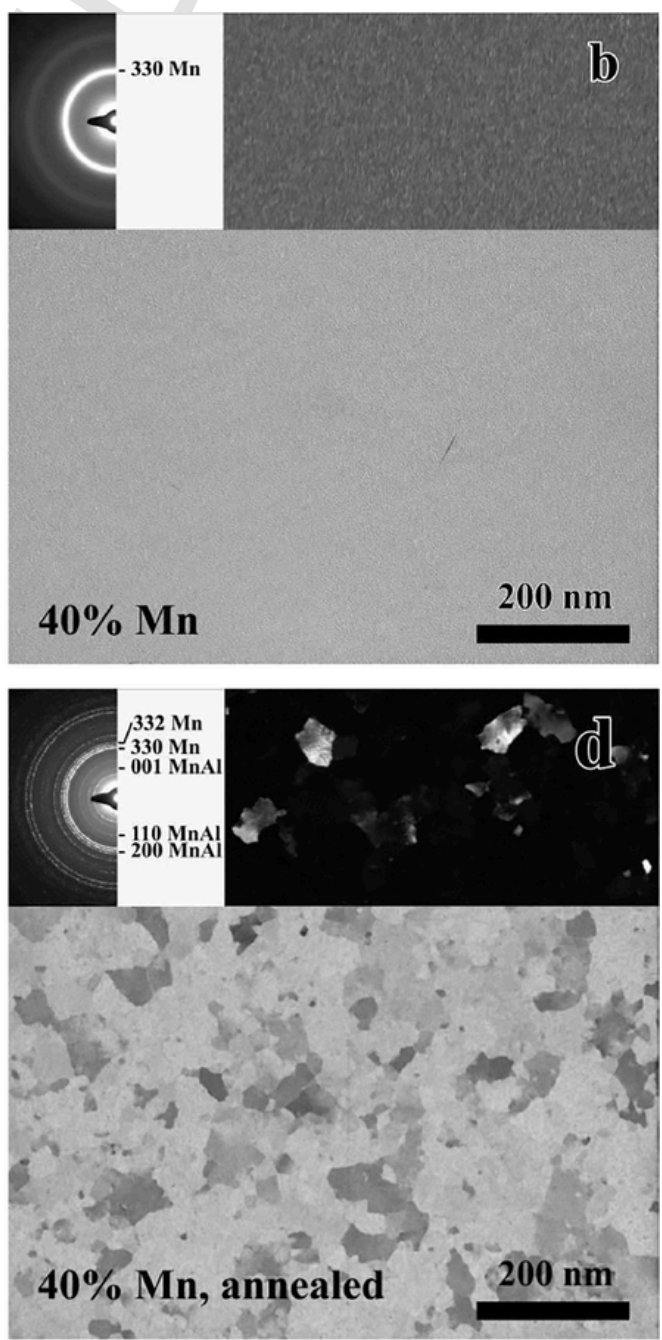

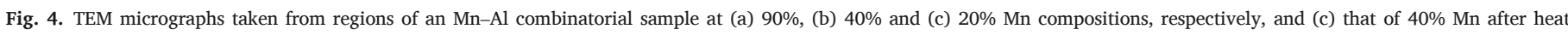
treatment at $500{ }^{\circ} \mathrm{C} / 0.5 \mathrm{~h}$ in $\mathrm{Ar}-\mathrm{H}_{2}$ mixture. The insets are SAED patterns and DF images. 
scanning AES. For that purpose, simultaneously with TEM grids Si substrates were used for deposition of various samples. Examples are given in Fig. 5. A MnAl $\mu$-combinatorial sample deposited on a $3 \mathrm{~mm}$ diameter Si disk is represented in (a). Except for the substrate, its build-up is identical to that of the sample shown in Fig. 2. The direction of the Mn-Al concentration gradient is marked with the arrow in Fig. 5(a). Thanks to the solid substrate such a sample is prime for concentration-dependent nano-indentation measurements.

Fig. 5(b) shows a TEM cross section of an Mn-Al layer that was prepared at $400{ }^{\circ} \mathrm{C}$ during a run for depositing a "regular" $\mu$-combinatorial sample, however, its substrate ( $\mathrm{Si}$ slab) was placed outside the combinatorial device. The slab was facing, uncovered, the magnetron sources, and as a result the layers of various compositions were deposited on top of each other. The concentration gradient of $\sim 0.14 \%$ per $n$ is perpendicular to the substrate as marked by the arrow in (b). This approach represents a simplified "one-sample concept" combinatorial technique that needs no special device, except two sources with controllable flux. Samples deposited this way are suitable for AES depth profiling, as well for study of diffusion. The layers of various compositions are densely packed, with a gradient about 2000 times higher compared to $\mu$-combinatorial TEM samples. Therefore, careful considerations are advised on inter-diffusion of the components, especially, at elevated temperatures.

To go on with examples of application, the adjustable compositional gradient of "one-sample concept" combinatory allows a scale up to sample sizes suitable for Ellipsometry, RBS, and XRD. Accordingly, $25 \times 12 \mathrm{~mm}^{2}$ size combinatorial $\mathrm{Si}-\mathrm{Ge}$ samples with $20 \mathrm{~mm}$ long gradient sections were deposited onto Si slabs, recently. The correlations of composition, structure and optical properties of SiGe layers will be published elsewhere.

Regarding TEM a specific advantage of $\mu$-combinatory is that the phases of the binary film are formed and investigated side by side, in a single TEM specimen that provides superior reproducibility and a straight comparison. The all-in-one feature makes $\mu$-combinatory very efficient; only one sample is to handle, there is no need for laborious TEM preparations, replacement and study of a series of samples. Furthermore, consistency, and tailored concentration profile of $\mu$-combinatorial samples unburden TEM characterization to a great extent and are an invitation to extremely high throughput automated TEM. That may be implemented by combining techniques similar to precession electron diffraction [14] and computer-controlled stage positioning.

\section{Conclusions}

Based on the "one-sample concept" a combinatorial method was developed for the preparation and study of gradient samples on single TEM grids. The sample is deposited through a slot that is moved over the grid, meanwhile, the powers of the DC magnetron sources are modulated so that to effectuate a compositional variation. The technique called " $\mu$-combinatory" was successfully demonstrated by the preparation, as well as, EDS and TEM characterization of an Mn-Al sample with linear concentration profile that covers the entire composition range. The microstructure of thin films is influenced by a number of deposition parameters. During $\mu$-combinatorial deposition the variable fluence ratio of the sources is transformed, by the moving slot, to a spatial variation of elemental composition at the TEM grid. It is plausible that, in addition to composition, the influence of any deposition parameter that can be varied with time (temperature, partial pressure of gases, additives, bias voltage, etc.) may be studied using $\mu$-combinatory. That is a potential to open up a range of applications. The main advantage of $\mu$-combinatory is that both fabrication and TEM characterization occurs as "all-in-one" i.e. a complete set of microstructural and analytical data of a binary system is condensed into a single gradient specimen. The "all-in-one" feature of $\mu$-combinatory and the potential for automation provide extremely high throughput for TEM, as well, other analytical techniques for solving a great many technological problems, exploring new materials and assembling phase libraries of binary films.

\section{Acknowledgments}

The technical contributions of N. Szász, J. Szívós and M. Német are highly acknowledged. The author thanks L.J. Lábár and M. Serényi for valuable discussions and careful readings of the manuscript.

This work was supported by the Hungarian Governmental Development and Innovation Program No.: GINOP 2.1.7-15.

\section{Supplementary materials}

Supplementary material associated with this article can be found, in the online version, at doi:10.1016/j.ultramic.2018.01.001.
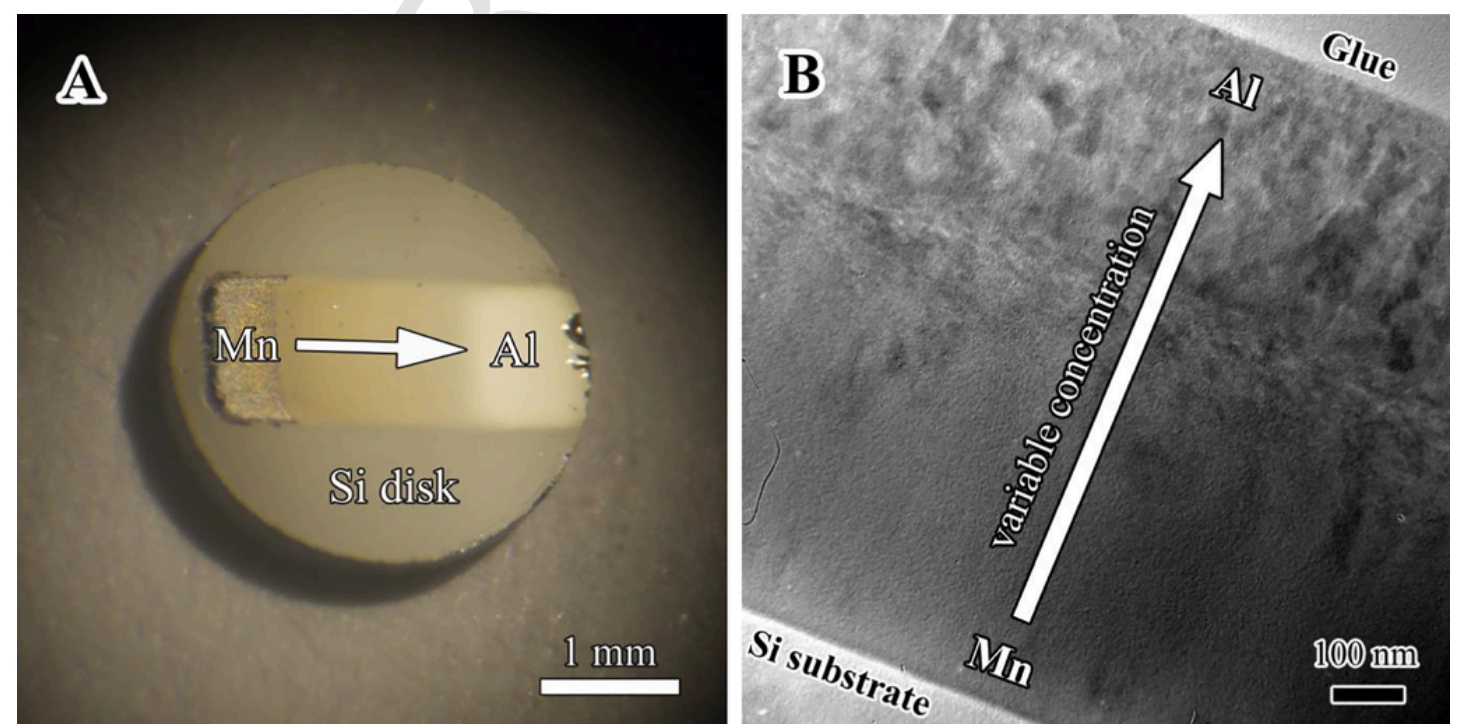

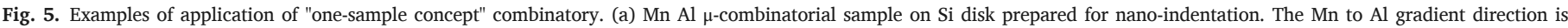
marked with the arrow. (b) TEM cross section of an Mn Al sample on Si uncovered during a $\mu$-combinatorial deposition run. The gradient is vertical, suitable for AES depth profiling. 


\section{References}

[1] Binary Alloy Phase Diagrams-Second edition. T.B. Massalski, Editor-in-Chief; H. Okamoto, P.R. Subramanian, L. Kacprzak,Editors. ASM International, Materials Park. Ohio. USA. December 1990 ISBN: 978-0-87170-403-0.

[2] J.J. Hanak, J. Mater. Sci 5 (1970) 964-971.

[3] X.D. Xiang, X. Sun, G. Briceño, Y. Lou, K.A. Wang, H. Chang, W.G. Wallace-Freedman, S.W. Chen, P.G. Schultz Sci 268 (5218) (1995) 1738-1740, Jun 23.

[4] K.E. Roskov, T.H. Epps, B.C. Berry, S.D. Hudson, M.S. Tureau, M.J. Fasolka, J. Comb. Chem. 10 (6) (2008) 966-973.

[5] P.D. Rack, J.D. Fowlkes, Y. Deng, Microsc. Microanal. 10 (S02) (August 2004) 58-59.
[6] D. Julthongpiput, W. Zhang, M.J. Fasolka, Microsc. Microanal. 10 (S02) (August 2004) 1108-1109.

[7] C.H. Olk, D.B. Haddad, J. Mater. Res. 21 (5) (May 2006).

[8] A. Kovacs, P.B. Barna, Solid State Ionics 141-142 (2001) 105

[9] F. Misják, P.B. Barna, A.L. Tóth, T. Ujvári, I. Bertóti, G. Radnóczi, Thin Solid Films 516 (2008) 3931-3934.

[10] F. Jiménez-Villacorta, J.L. Marion, T. Sepehrifar, M. Daniil, M.A. Willard, L.H. Lewis, Appl. Phys. Lett. 100 (2012), 112408.

[11] G. Sáfrán Hung. Patent No. P1500500 (2015)

[12] L. Tóth, Á. Barna, G. Sáfrán, M. Menyhárd, T. Korányi, VACUUM 33 (1-2) (1983) 111.

[13] J.L. Lábár, Ultramicroscopy 103 (2005) 237-249.

[14] A.S. Eggeman, P.A. Midgley, Precession electron diffraction, P.W. Hawkes Advances in Imaging and Electron Physics., 170, 20121-63. 